\title{
Patterns of adherence behaviour for patients with glaucoma
}

H Cate ${ }^{1,2}$, D Bhattacharya ${ }^{2}$, A Clark ${ }^{3}$, R Holland ${ }^{3}$ and DC Broadway ${ }^{1}$

\begin{abstract}
Aims and purpose Adherence to long-term treatment regimens for primary open-angle glaucoma holds a challenge for both clinicians and patients. The study aims were to (i) establish the magnitude of travoprost non-adherence using an Electronic Adherence Monitor (EAM), (ii) compare electronic with patient self-reported adherence, and (iii) explore the application of a previously reported method of graphically presenting adherence data to a larger cohort over a longer monitoring period.

Methods A cohort study of patients using travoprost for glaucoma or ocular hypertension was conducted. All participants used an EAM and adherence data were collected prospectively for 2 months. Selfreported adherence was obtained using the Morisky Medication Adherence Scale (MMAS); patients also reported frequency of missed doses. Potential predictors of adherence were collected via a structured interview. EAM-recorded interdose intervals were plotted graphically.

Results Of 100 patients invited to participate, 98 consented and EAM data were collected successfully from 88 participants. The median EAM adherence score for the cohort was $88.9 \%$ (interquartile range: 71.2 , 92.2). When dichotomised ( $\geq 80 \%$ : adherent; $<80 \%$ : non-adherent), EAM identified $36.7 \%$ as non-adherent and MMAS $\mathbf{1 2 . 2 \%}$. EAM data were used to classify five types of adherence behaviour including a category representing levels of $\geq 97 \%$ maintained by $21 \%$ of participants.

Conclusion EAM revealed good adherence to glaucoma monotherapy but poor agreement with patient self-reported adherence. An adherence category of persistent and exceptionally high adherence
\end{abstract}

to travoprost over a 2-month period was identified.

Eye (2013) 27, 545-553; doi:10.1038/eye.2012.294; published online 18 January 2013

Keywords: glaucoma; adherence; compliance; Travatan Dosing Aid; electronic monitoring

\section{Introduction}

Primary open-angle glaucoma (POAG), due to its slow, progressive and initial asymptomatic nature, holds particular challenges to maintaining long-term patient adherence to therapy. Intraocular pressure (IOP) reduction with ocular hypotensives is effective in preventing glaucoma progression; thus, nonadherence is a risk factor for optic neuropathy. ${ }^{1}$ Early identification of patient nonadherence by clinicians and implementation of appropriate interventions therefore is essential. However, several studies have shown that clinicians are unable to distinguish between adherent and non-adherent patients using IOP or other subjective markers. ${ }^{2-4}$ One good IOP measure within the target range does not constitute good adherence nor does a higher than expected reading equate to poor adherence as treatment efficacy, type of glaucoma, and frequency of missed doses (FMDs) must all be considered. ${ }^{5}$ Therefore, it can be difficult for clinicians to establish true efficacy of prescribed ocular hypotensives. With poor clinical response, a change to therapy is often initiated, potentially exacerbating the problem since adherence declines with increasing regimen complexity. ${ }^{6-8}$

Discussing adherence is a sensitive subject and thus it is challenging to obtain an honest
${ }^{1}$ Eye Department, Norfolk and Norwich University Hospital, NHS Foundation Trust, Norwich, UK

${ }^{2}$ School of Pharmacy, University of East Anglia, Norwich, UK

${ }^{3}$ Norwich Medical School, University of East Anglia, Norwich, UK

Correspondence: H Cate, Eye Department, Norfolk and Norwich University Hospital, NHS Foundation Trust, Colney Lane, Norfolk, Norwich NR4 7UZ, UK Tel: + 44 (0)1603 288870; Fax: + 44 (0)1603 288261. E-mail: heidi.cate@nnuh. nhs.uk

Received: 15 October 2012 Accepted in revised form: 17 December 2012 Published online: 18 January 2013 
response. In one study, $69 \%$ of patients prescribed ocular hypotensive medication, when asked, did not reveal their poor adherence or persistence. ${ }^{6}$ The use of validated patient self-report adherence measures may therefore provide a more accurate indication. Few self-report measures are bespoke for ocular medication; the most widely used is the Morisky Medication Adherence Scale (MMAS), which has been validated for use with patients prescribed oral medication for the treatment of hypertension. ${ }^{9}$ If effective for ocular medication, such a self-report measure offers a relatively cheap and quick method of adherence estimation by clinicians within routine practice.

Reported adherence rates from glaucoma studies have varied widely from 5 to $80 \%{ }^{10}$ The variation may be partly attributable to inconsistency in the definition of non-adherence. An $80 \%$ adherence rate is widely recognised as 'acceptable' for many systemic medications, ${ }^{11}$ but there is no such acceptance for ocular hypotensives. To be clinically relevant, an 'acceptable' adherence level should be determined by its impact on clinical outcome. ${ }^{10}$ Such evidence is lacking for ocular hypotensives due to the requirement for long-term follow-up and known inaccuracies in determining IOP control, $^{12}$ visual field defects, or optic nerve damage. However, a recent cross-sectional glaucoma study in the United States found that participants with adherence rates $<80 \%$, according to a medication event monitoring system (MEMS), had worse visual field defects than those with adherence rates $\geq 80 \%$. $^{13}$

The wide variation in reported adherence rates may also be a product of subjectivity in the self-report adherence assessment methods frequently employed. The Travatan Dosing Aid (TDA) (Travalert; Alcon Inc., Fort Worth, TX, USA) was designed for use with Travatan (travoprost) or Duotrav (travoprost/timolol combination) and electronically stores the time, date, and number of drops administered. The TDA therefore provides an objective measure of adherence. Studies using the TDA have reported accurate recording of drop administration ${ }^{2,14,15}$ and adherence rates from 60 to $>80 \% .2,16,17$ Routine use of electronic adherence monitoring in clinical practice is not yet practical because of the prohibitive costs. Only one of these studies explored the relationship between self-reported adherence and the TDA, ${ }^{2}$ and reported a poor association between self-report and TDA-identified non-adherence; however, a validated self-report adherence measure was not used. A clear need remains for determining whether a validated self-report measure may provide an accurate indication of non-adherence.

A further limitation of the existing studies is that participants have been newly initiated onto the ocular hypotensive therapy being monitored and thus the effect of duration of therapy on adherence was not explored. Studies with other medical conditions have demonstrated a decline in adherence with time. ${ }^{18-20}$

In addition to simply quantifying non-adherence, describing its patterns and relationships with other measureable parameters may further our understanding and aid intervention design.

Ajit et $a l^{17}$ graphically presented interdose intervals recorded by the TDA, which resulted in categorisation of four adherence behaviour patterns. This study allowed different adherence patterns to travoprost therapy to be elucidated but was limited by a sample size of 37 participants.

There is therefore a need for adherence patterns to topical ocular hypotensive therapy to be explored in a larger population. Given the wide variation in reported adherence rates with ocular hypotensives and that these are largely reported for patients newly initiated on therapy, ${ }^{10}$ a better estimate of non-adherence magnitude in the wider population may be obtained by determining whether magnitude is influenced by the duration of prescribed therapy. Furthermore, the relationship between TDA and validated self-reported adherence measure data needs establishing to ascertain whether it may be appropriate for non-adherence identification in routine clinical practice.

\section{Materials and methods}

A 2-month cohort study of patients attending a glaucoma clinic in a UK teaching hospital was conducted.

\section{Participant selection and recruitment}

Patients with POAG or ocular hypertension and treated with travoprost monotherapy were eligible for study participation. Patients were excluded if unable to provide informed consent or reliant upon paid carers to manage their medication. Eligible patients were informed of the study during their clinic consultation and approached a researcher if interested in participation.

Sample size and group allocation A sample size of 100 participants was deemed feasible for recruitment within a 12-month period. Experience of travoprost use was recorded to recruit 50 participants to each of the following two groups:

- Follow-up group: Already using travoprost and thus experienced drop users.

- Newly prescribed group: Initiated onto travoprost at the time of recruitment and thus drop naïve. 


\section{Data collection}

The TDA is designed to assist drop administration and can be replenished with a new travoprost bottle by patients. The TDA is equipped with an audible beep and visual reminder symbol on its display, which were inactivated to enable adherence under usual clinical care conditions to be measured. Participants were given a demonstration of the TDA before commencing use of it for approximately 8 weeks.

Information about baseline sociodemographic characteristics and the following possible predictors of adherence were collected using a structured interview; a positive family (parent, sibling, or offspring) history of glaucoma, experience of problems using drops, use of other prescribed medication and if this was used at the same time as travoprost, and self-administration of drops or help given by a family member.

After 8 weeks, participants were provided with a questionnaire designed to assess patient medicationtaking behaviour and potential predictors of adherence. Self-reported adherence was determined by two approaches: Morisky Medication Adherence Scale $(\mathrm{MMAS})^{9}$ and patient reported FMDs. The MMAS measure has been shown to have $69 \%$ accuracy but only $44 \%$ sensitivity, and has been correlated with positive clinical outcomes in systemic hypertension studies. ${ }^{9}$ The FMD was devised by the authors in order for participants to quantify their self-estimated non-adherence in terms of missed doses. In addition, the TDA was retrieved and data extracted.

\section{Analysis}

Descriptive statistics were used to characterise the total participant population and compare 'newly prescribed' with 'follow-up' participants. The TDA has been reported to make extra, erroneous recordings, ${ }^{15}$ so regardless of uni- or bi-lateral dosing instruction, only one lever depression was required each day to register a positive recording. A formula was devised to calculate the mean dosing time over the monitoring period. Drops were prescribed for once-daily evening use; thus, an adherent event had to fall within $\pm 2 \mathrm{~h}$ of the calculated mean dosing time, between 1700 and 0500 hours.

Percentage adherence for the sample population was reported as a mean plus $95 \%$ confidence interval $(95 \% \mathrm{CI})$ or median plus interquartile range (IQR), as appropriate, depending on the data distribution. A linear-by-linear association test was used to establish predictors of adherence behaviour. To allow comparison with binary MMAS and FMD data, TDA data were dichotomised to 'adherent' if the average number of recorded doses was $\geq 80 \%$ of expected and 'non-adherent' if $<80 \%$. TDA adherence rates for 'newly prescribed' and 'follow-up' participants were also reported and compared using an independent Student's $t$-test or Mann-Whitney U-test depending on the data distribution. A $\chi^{2}$ test was used to compare the MMAS and FMD adherence rates between the newly treated and follow-up groups.

The travoprost dosing intervals recorded by the TDA were plotted to graphically describe participant dosing behaviour. The behaviours were categorised using the four categories described by Ajit et al: ${ }^{17}$ Type 1 discontinued after a relatively short dosing period; Type 2 had an adherence rate $>80 \%$; Type 3 had frequent drug holidays; and Type 4 had more variable frequent missed doses with adherence often below $60 \%$.

The MMAS is composed of four 'yes/no' questions about past medication use patterns. Participants answering 'yes' to a question score ' 1 '; thus, scores were cumulative and ranged from 0 to 4 . Participants were dichotomised according to score: $0=$ adherent; $1-4=$ non-adherent. The FMD required participants to tick one option that best quantified the number of missed doses ranging from none, 1, 2-3, 4-9, 10-19, or $\geq 20$ per month. Participants were dichotomised according to their response: $\geq 80 \%$ doses taken $(<3$ doses missed per month $)=$ adherent; $<80 \%$ doses taken $(\geq 4-9$ doses missed per month $=$ non-adherent. For both MMAS and FMD, the percentage of adherent participants (95\% CI) was reported. Sensitivity and specificity relative to TDA and MMAS were measured.

The participant population and magnitude of adherence were characterised using descriptive analysis. Cohen's kappa was used to measure agreement between the dichotomised adherence scores of the TDA $(\geq 80 \%=$ adherent; $<80 \%=$ non-adherent) with MMAS and FMD. Mann-Whitney $U$-test was used to establish any difference between mean adherence measured by the TDA and the adherent and non-adherent groups measured by MMAS and FMD.

Logistic regression was used to identify possible adherence predictors; univariate models were used to estimate the odds ratios, $95 \%$ CIs, and $P$-values. If appropriate, a multivariate model was constructed to identify independent predictors of adherence.

The study received ethical approval from Norfolk Research Ethics Committee and all applicable institutional and governmental regulations concerning the ethical use of human volunteers were followed during the research.

\section{Results}

The study recruitment period was January to December 2009. Of 100 patients invited, 98 consented: 49 were 'follow-up' group participants and 49 'newly prescribed'. 
Table 1 summarises the demographic characteristics of the sample population. In addition to the difference in duration of prescribed travoprost, the 'follow-up' group had a greater proportion of people with significant previous/current medical conditions. The 'follow-up' and 'newly prescribed' groups were comparable with respect to age and gender; however, the 'newly prescribed' group had a greater proportion of participants who left school at 16 years of age.

TDA data were successfully collected from 88 (89.8\%) participants: 43 (49\%) from the 'follow-up' group and $45(51 \%)$ from the 'newly prescribed' group. Of the remaining 10 participants, two did not return the TDA, four chose not to use the TDA, one had treatment stopped, one had a malfunctioning TDA, and two were not contactable.

The median adherence measured by the TDA for the 'newly prescribed' group was $89.1 \%$ (IQR: 71.6, 98.1) compared with $85.7 \%$ (IQR: 55.9, 94.6) for the 'follow-up' group. Mann-Whitney $U$-test demonstrated no significant difference $(P=0.198)$. MMAS identified 2 $(4.7 \%$; $95 \%$ CI: $3.8,5.6)$ to be non-adherent in the 'newly prescribed' group and 8 (20.5\%; 95\% CI:18.5, 22.5) in the 'follow-up' group. A test of significance was not appropriate because of insufficient non-adherent numbers in the 'newly prescribed' group. No participants reported non-adherence using FMD. There was no significant difference in adherence between the newly treated and follow-up group; thus, further analysis was carried out with the groups as one collective cohort.

Median adherence measured by the TDA was $86.89 \%$ (IQR: $60.8,96.2)$ and $40.9 \%$ (95\% CI: 26.5, 46.9) of participants were recorded with $<80 \%$ adherence. MMAS and TDA data were available for 82 participants and the MMAS identified $10(12.2 \%$; 95\% CI: 5.1, 19.3) to be non-adherent.

A comparison of the dichotomised TDA data with MMAS $(n=75)$ demonstrated $86 \%(95 \%$ CI: 78, 94) sensitivity and $17 \%$ (95\% CI: 9, 25) specificity. As no participants reported missing $<80 \%$ of doses per month using FMD, no further analysis was undertaken. Figure 1 provides a comparison of TDA adherence with MMAS and illustrates higher self-reported adherence relative to TDA records. Cohen's kappa did not identify significant agreement between MMAS and TDA adherence ( -0.056 , $P=0.525)$. There was no significant difference in TDA measured adherence between MMAS adherent and nonadherent groups (Mann-Whitney $U$-test, $P=0.189$ ).

Both uni- and multivariate models found no demographic characteristics to be significant predictors of adherence.

Figure 2 presents examples of five different adherence behaviour patterns from the TDA data. These were derived from 88 participants for whom data were available. The four patterns of classification described by Ajit et $a l^{17}$ plus an additional category (Type 2) to differentiate between participants with acceptable adherence $(>80 \%)$ and those with excellent adherence $(\geq 97 \%)$ are illustrated.

A summary of participant demographics and potential predictors of adherence for each category of adherence behaviour are shown in Table 2. A test of trend between Type (Type 2, Type 3, and the combined score

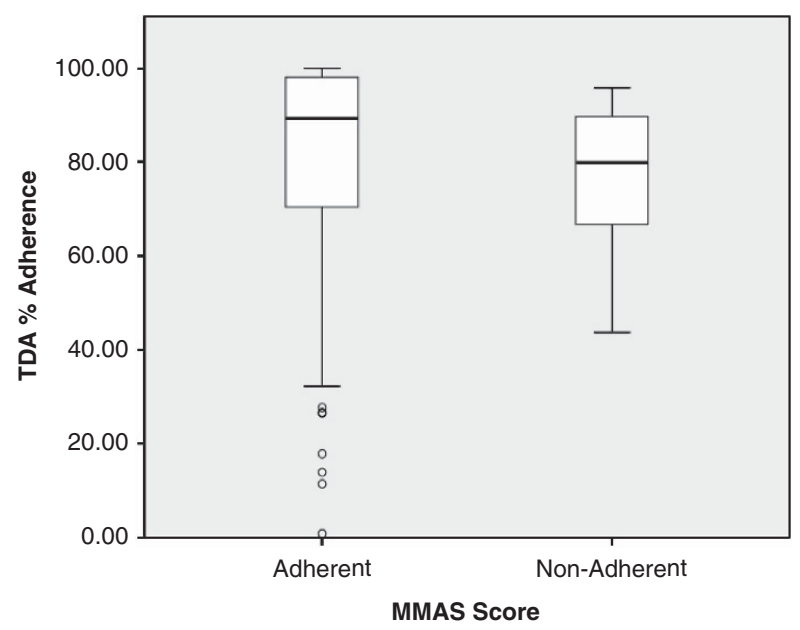

Figure 1 A comparison of Morisky Measures of Adherence with Travalert Dosing Aid \% adherence.

Table 1 Population demographics at baseline

\begin{tabular}{|c|c|c|c|}
\hline Characteristic & Total cohort & Follow-up group & Newly prescribed group \\
\hline No. $(\%)$ male $(n=98)$ & $50(51 \%)$ & $21(43 \%)$ & $29(59 \%)$ \\
\hline Median age, years (IQR) $(n=98)$ & $72(63,78)$ & $72(63,78)$ & $70(62,78)$ \\
\hline No. $(\%)$ employed $(n=92)$ & $67(72.8 \%)$ & $32(71 \%)$ & $35(75 \%)$ \\
\hline No. $(\%)$ British $(n=97)$ & $94(96.9 \%)$ & $48(100 \%)$ & $46(94 \%)$ \\
\hline No. $(\%)$ married/partner $(n=91)$ & $67(73.6 \%)$ & $32(70 \%)$ & $35(78 \%)$ \\
\hline No. $(\%)$ left school at 16 years $(n=90)$ & $50(55.6 \%)$ & $19(43 \%)$ & $31(67 \%)$ \\
\hline No. $(\%)$ with family members with glaucoma $(n=79)$ & $25(68.4 \%)$ & $12(32 \%)$ & $13(31 \%)$ \\
\hline No. $(\%)$ with a medical condition $(n=91)$ & $58(63.7 \%)$ & $33(73 \%)$ & $25(54 \%)$ \\
\hline Median duration of travoprost use, days (IQR) $(n=98)$ & $71(0,535)$ & $532(197,918)$ & 0 \\
\hline
\end{tabular}




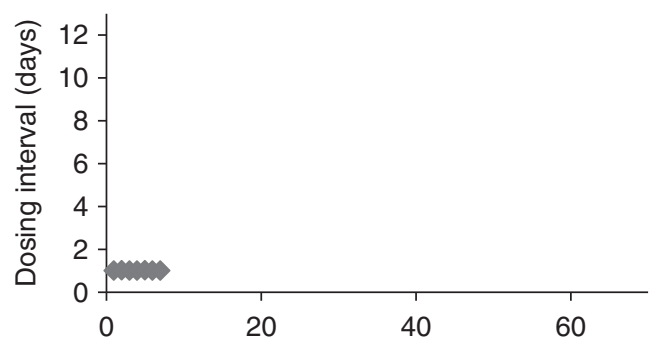

\section{Type 1 Discontinued after a short time}

Demonstrated by $9(10 \%)$ of participants.

This participant stopped after 7 days.

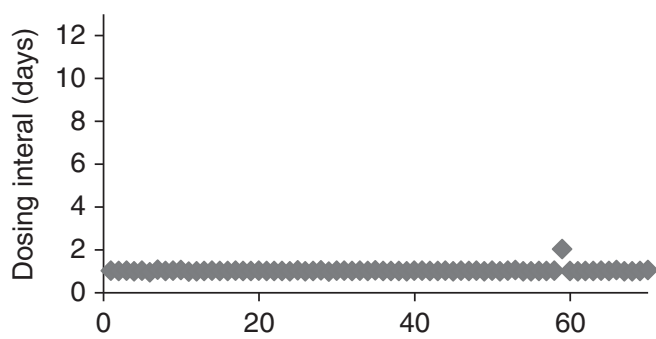

Type 2 Adherence $\geq 97 \%$

(No more than 2 missed doses for more than 2 days).

Demonstrated by $18(21 \%)$

of participants.

This participant has an adherence rate of $99 \%$.

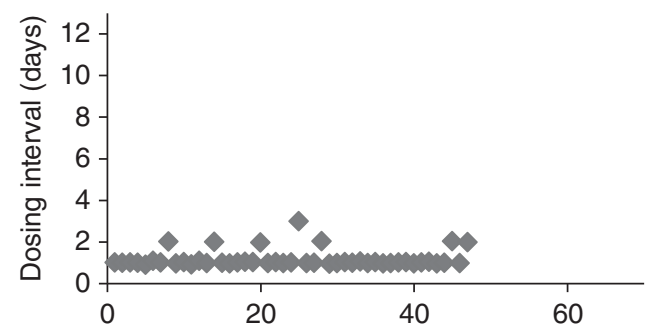

Type 3 Adherence $<97>80 \%$ and more variable than Type 2

Demonstrated by 31 (35\%) of participants.

This participant has an adherence rate of $84 \%$.

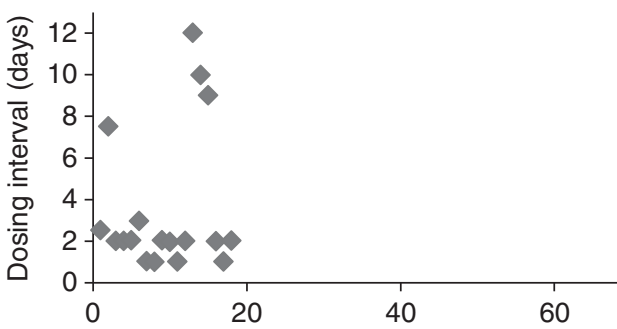

Type 4 Adherence $<80 \%$ with frequent drug holidays (7 days without dosing)

Demonstrated by 9 (10\%) of participants.

This participant has an adherence rate of $28 \%$.

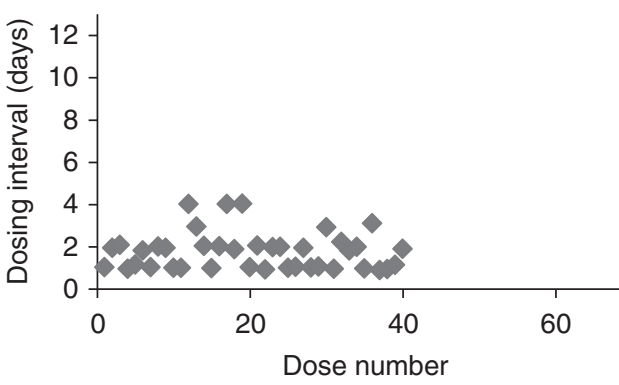

Type 5 Adherence $<80 \%$ with variable and frequent missed doses

Demonstrated by 21 (24\%) of participants.

This participant has an adherence rate of $51 \%$.

Figure 2 Graphical representation of adherence behaviours.

of Types 4 and 5) revealed no statistically significant predictors for type of adherence behaviour.

\section{Discussion}

The high consent rate and cohort demographic characteristics, which are representative of the wider population with glaucoma, afford some confidence in the generalisability of the reported findings. The absence of a relationship between adherence and sociodemographic factors supports previous findings $2,17,21,22$ and the rationale not to stratify participant randomisation based on sociodemographic factors. 
Table 2 Summary of participant demographics for each type of adherence behaviour

\begin{tabular}{|c|c|c|c|c|c|c|}
\hline \multirow[t]{3}{*}{ Characteristic } & \multirow{3}{*}{$\begin{array}{l}\text { Total } \\
\text { cohort }\end{array}$} & \multirow{2}{*}{$\begin{array}{c}\text { Discontinued } \\
\text { after } \\
\text { a short time }\end{array}$} & \multicolumn{4}{|c|}{ Adherence } \\
\hline & & & $\geq 97 \%$ & $\begin{array}{l}<97 \% \\
\geq 80 \%\end{array}$ & $\begin{array}{l}<80 \% \text { with drug } \\
\text { holidays }\end{array}$ & $\begin{array}{l}<80 \% \text { with } \\
\text { variable dosing }\end{array}$ \\
\hline & & Type 1 & Type 2 & Type 3 & Type 4 & Type 5 \\
\hline No. $(\%)$ Male $(n=88)$ & $46(53 \%)$ & $6(67 \%)$ & $9(53 \%)$ & $15(50 \%)$ & $7(78 \%)$ & $9(45 \%)$ \\
\hline Median age, years (IQR) $(n=88)$ & $72(63,78)$ & $68(64,68)$ & $74(71,79)$ & $72(65,78)$ & $73(67,78)$ & $72(63,78)$ \\
\hline No. $(\%)$ employed $(n=82)$ & $21(27 \%)$ & $3(33 \%)$ & $5(29 \%)$ & $1(3 \%)$ & $4(44 \%)$ & $8(40 \%)$ \\
\hline No. $(\%)$ married/partner $(n=81)$ & $59(73 \%)$ & $5(56 \%)$ & $11(65 \%)$ & $25(83 \%)$ & $5(56 \%)$ & $13(65 \%)$ \\
\hline No. $(\%)$ left school at 16 years $(n=80)$ & $43(54 \%)$ & $4(44 \%)$ & $10(59 \%)$ & $17(57 \%)$ & $3(33 \%)$ & $9(45 \%)$ \\
\hline $\begin{array}{l}\text { No. }(\%) \text { with family members with glaucoma } \\
(n=70)\end{array}$ & $48(67 \%)$ & $1(11 \%)$ & $2(6 \%)$ & $9(30 \%)$ & $2(22 \%)$ & $9(45 \%)$ \\
\hline No. $(\%)$ uses other medication $(n=76)$ & $62(82 \%)$ & $6(67 \%)$ & $14(82 \%)$ & $20(67 \%)$ & $8(89 \%)$ & $14(70 \%)$ \\
\hline $\begin{array}{l}\text { No. }(\%) \text { uses other medication at same time as } \\
\text { travoprost }(n=62)\end{array}$ & $14(23 \%)$ & $1(11 \%)$ & $3(18 \%)$ & $6(20 \%)$ & $1(11 \%)$ & $3(15 \%)$ \\
\hline $\begin{array}{l}\text { No. }(\%) \text { encountered problems using } \\
\text { travoprost }(n=75)\end{array}$ & $27(36 \%)$ & $4(44 \%)$ & $3(18 \%)$ & $10(33 \%)$ & $5(56 \%)$ & $5(25 \%)$ \\
\hline $\begin{array}{l}\text { No. }(\%) \text { needed help to administer travoprost } \\
(n=76)\end{array}$ & $20(26 \%)$ & $2(22 \%)$ & $5(29 \%)$ & $7(23 \%)$ & $2(22 \%)$ & $4(20 \%)$ \\
\hline No. $(\%)$ sought further advice $(n=75)$ & $24(32 \%)$ & $2(22 \%)$ & $3(18 \%)$ & $37(17 \%)$ & $2(22 \%)$ & $6(30 \%)$ \\
\hline
\end{tabular}

The adherence rate was higher than expected in comparison with other studies using the TDA, 2,16,17 which may be attributable to a number of reasons such as reactivity bias and/or monitoring duration. ${ }^{23,24} \mathrm{In}$ addition, TDA data were missing for 10 participants: two for reasons beyond the participant's control and eight due to participant choice. It is possible that poorer adherers may have been more inclined to choose not to allow their adherence to be monitored using the TDA and thus the reported adherence estimate may have been inflated. Although it is unlikely that eight participants from a cohort of 98 would skew the data, their pattern of adherence is missing from the analysis described in Figure 2.

It has been reported that 9 out of 10 patients are unable to instil their drops correctly. ${ }^{25}$ While the TDA is an objective measure of adherence, it assumes that the eye drop was always successfully administered; when all that can really be inferred is that the patient attempted a dosing event at a specified time. Attempting to observe directly treatment application to confirm a connection between TDA data and actual adherence is problematic because of the participant-researcher interaction profoundly altering participant behaviour. ${ }^{26}$ Participant awareness of adherence monitoring and consenting to study participation may themselves have produced Hawthorne effects causing participants to be more adherent to their medication regimen. The Hawthorne effects found in studies measuring adherence to glaucoma medication have been documented previously. ${ }^{2,8}$ When research assessment prepares people to be more receptive to certain behaviours than would be the case in the absence of research assessment, a synergistic relationship occurs, which may influence the study observations. ${ }^{23}$

The absence of a significant difference in adherence magnitude between 'newly prescribed' and 'follow-up' participants contradicts adherence studies involving other clinical conditions, which have demonstrated a decline in adherence with time. This may be due to the low prevalence of non-adherence, resulting in a sample size insufficient to identify any difference.

This study also demonstrated the expected inconsistency in self-reported adherence relative to TDA. Participants identified by the TDA to be $\leq 80 \%$ adherent largely self-reported perfect adherence, and thus reinforcing previous evidence that self-report measures yield higher adherence estimates. 2,17,27 The discrepancy between self-report and objective measures of adherence is often attributed both to the social desirability to be adherent to medication regimens and memory bias. Unintentional non-adherence is a passive behaviour often considered as forgetfulness or confusion; therefore, it is unsurprising that overestimation of medication use may arise from such behaviours as these cannot be reported if the dose event has been forgotten or misinterpreted. The failure of the 'frequency of missed dose' method to capture self-report of non-adherence might therefore have been due to measurement error of the tool. To report frequency of non-adherence, the respondent must retrieve this information from their long-term memory. However, commonly occurring events are harder to distinguish and recall individually. ${ }^{28}$ Cognitive heuristics are often employed by respondents 
when faced with frequency questions of incomplete memory events. The number of items to be recalled will determine whether a counting or estimating strategy is used by the respondent. ${ }^{29}$

Reporting adherence rates alone provides limited information about patient behaviour, whereas categorisation of the types of non-adherent behaviour identified by the TDA improves our understanding of the potential sources of non-adherence and gives relevance to clinical practice.

Participants demonstrating $>80 \%$ adherence, first reported by Ajit et al. ${ }^{17}$ as Type 2 behaviour, represented the majority of participant behaviour in this study and thus warranted further subdivision (Type 2 and Type 3 behaviours). The absence of consensus regarding the minimum necessary adherence with glaucoma preparations provides further rationale for examining this cohort in greater detail.

For the remainder of the cohort exhibiting poor adherence, some inferences can be made regarding the cause. Type 1 participants, who stopped using travoprost early, may have done so because of travoprost-induced side effects and thus sought medical advice, which resulted in treatment termination. Participants displaying Type 4 behaviour (dosing holidays) are of concern with respect to glaucoma management, due to the number of days without adequate treatment to control IOP.27 However, ocular hypotensive agents not only lower IOP but also minimise IOP fluctuations (a potential risk factor for glaucomatous deterioration), 1,30 strengthening the claim that patients with glaucoma should aim for $100 \%$ adherence to reduce such fluctuations in IOP. Thus, participants displaying Type 5 behaviour with variable missed doses are arguably at greater risk of developing progressive glaucomatous visual loss. The increased peaks and troughs of IOP, synthetically created through the frequent stopping and starting of treatment, may inadvertently magnify the very fluctuations that prostaglandins aim to remove. The complexity and ethical implications associated with such a theory obstructs the collection of empirical evidence. In general, minor and sporadic dosing errors seen in Type 5 behaviours are more difficult to detect as they are unintentional and often erratic, when compared with Type 4, repeated dosage errors that stem from intentional behaviour traits. ${ }^{31}$

To improve our understanding of non-adherent behaviour, a larger sample size may enable mapping of self-reported non-adherence with observed adherence patterns to find unintentional non-adherence. It has been suggested that multiple self-report tools would be required to detect all the different types of nonadherence. ${ }^{31}$ Determination of non-adherence subtype might be particularly relevant with respect to topical ocular medications due to the added complexity of administering drops from a bottle which may not only be difficult to achieve but also restricts the ability to use the packaging as a reminder. In contrast, patients report that when using tablets from a 'blister pack', the empty 'blister' can act as a visual reminder that the intended dose has been administered, or tablets can be transferred into dosing boxes to aid the memory. Such visual cues or practical remedies are not possible with a bottle of drops.

Given the different reasons for suspected nonadherence and likely demand for different remedial intervention strategies, it is important that clinicians identify non-adherence and establish the reason(s). ${ }^{32} \mathrm{No}$ predictors to aid early detection of patients likely to be non-adherent were identified in this study. Thus, discussion between patient and clinician is essential, as recommended by UK national guidelines, in all cases. ${ }^{33}$ Patients who are not confident with drop administration or have manual dexterity disabilities may require the use of a dosing aid. Patients who struggle with forgetfulness may require an electronic dosing reminder or help to establish use of drops as part of a daily routine. It is also important to appreciate that while patients are asked to use travoprost every evening to achieve greater daytime efficacy and a narrower range of 24-h pressure fluctuation, ${ }^{1}$ some data have indicated that the effects of travoprost may last as long as $84 \mathrm{~h}$ postdose. ${ }^{34,35}$ Thus, the frequency of dosing may be less imperative for patients using travoprost, although until the consequences of nonadherence and the intervals between dosing of travoprost (or any ocular hypotensive) are established, clinicians advocate a once-daily evening application of travoprost. Clinicians may also consider giving more education about drop application techniques and consequences of nonadherence or simplifying dosing regimens. ${ }^{36}$

If following discussion with the patient, long-term adherence to eye drop therapy is not feasible, selective laser trabeculoplasty (SLT) $29,37,38$ may be an option as it is less invasive than incisional surgical options. However, it should be acknowledged that SLT is not universally efficacious or available and many patients have a preference for topical medication.

The risks associated with poor adherence are well documented; however, predictors of non-adherence have been elusive and as expected, none were identified from basic demographic data. No significant decline in adherence with duration of prescribing was identified in this study; however, further work with a larger sample size is necessary to confirm this finding. Information regarding the magnitude and types of non-adherence to glaucoma therapy are limited; thus, adherence interventions grounded in evidence are lacking. This study demonstrated the magnitude of non-adherence and the type of non-adherent behaviour observed with travoprost and provides an evidence based on which to 
design future interventions to improve adherence. Application of this categorisation approach to describe adherence behaviour with other medicines for the treatment of long-term conditions may be appropriate. Further work is necessary to aid health-care practitioners in eliciting information from patients about their adherence behaviour. A better understanding of nonadherence will enable the provision of appropriate support to be focused on vulnerable individuals.

\section{Summary}

What was known before

- Adherence to glaucoma medication is poor and the variation of patient behaviour to medication use has only been reported from small cohorts.

- Understanding patient adherence behaviour helps healthcare professionals to improve adherence to glaucoma medication.

What this study adds

- An improved estimate of adherence magnitude and behaviour patterns of patients treated with eye drops.

- An indication of the effect of treatment duration on adherence.

- Further evidence that patients overestimate adherence.

- Evidence that certain patients display exceptional adherence to glaucoma monotherapy.

- Non-adherent patients remain difficult to identify in clinical practice; simple sociodemographic characteristics are inappropriate.

\section{Conflict of interest}

The authors declare no conflict of interest.

\section{Acknowledgements}

We thank Sally Edmunds, Donna Fisher, Corinne Haynes, Karen Le Grys and Naomi Waller, for their work collecting data and Tony Dyer (Norfolk Clinical Trials Unit) for designing the software programme to calculate adherence. We also thank Barbara Talbot and Michael Cudby for their contribution to the project as patient and public representatives.

\section{References}

1 Lee PP, Walt JW, Rosenblatt LC, Siegartel LR, Stern LS. Association between intraocular pressure variation and glaucoma progression: data from a United States chart review. Am J Ophthalmol 2007; 144: 901-907.

2 Okeke CO, Quigley HA, Jampel HD, Ying GS, Plyler RJ, Jiang $\mathrm{Y}$ et al. Adherence with topical glaucoma medication monitored electronically the Travatan Dosing Aid study. Ophthalmology 2009; 116: 191-199.

3 Kass MA, Gordon M, Meltzer DW. Can ophthalmologists correctly identify patients defaulting from pilocarpine therapy? Am J Ophthalmol 1986; 101: 524-530

4 Kass MA, Gordon M, Morley Jr., RE, Meltzer DW, Goldberg JJ. Compliance with topical timolol treatment. Am J Ophthalmol 1987; 103: 188-193.

5 Cate H, Broadway DC. Association between intraocular pressure and adherence: is there one? Eye (London) 2011; 25: 1238-1239

6 Winfield AJ, Jessiman D, Williams A, Esakowitz L. A study of the causes of non-compliance by patients prescribed eyedrops. Br J Ophthalmol 1990; 74: 477-480.

7 Sleath B, Robin AL, Covert D, Byrd JE, Tudor G, Svarstad B. Patient-reported behavior and problems in using glaucoma medications. Ophthalmology 2006; 113: 431-436.

8 Robin AL, Novack GD, Covert DW, Crockett RS, Marcic TS. Adherence in glaucoma: objective measurements of oncedaily and adjunctive medication use. Am J Ophthalmol 2007; 144: 533-540.

9 Morisky DE, Green LW, Levine DM. Concurrent and predictive validity of a self-reported measure of medication adherence. Med Care 1986; 24: 67-74.

10 Olthoff CM, Schouten JS, van de Borne BW, Webers CA. Noncompliance with ocular hypotensive treatment in patients with glaucoma or ocular hypertension an evidencebased review. Ophthalmology 2005; 112: 953-961.

11 Sackett D, Snow J. Magnitude and measurement of compliance. In: Haynes B, Wayne TD, Sacket D (eds) Compliance in Healthcare. The John Hopkins University Press: Baltimore and London, 1979.

12 Kulkarni SV, Damji KF, Buys YM. Medical management of primary open-angle glaucoma: best practices associated with enhanced patient compliance and persistency. Patient Prefer Adherence 2008; 2: 303-314.

13 Sleath B, Blalock S, Covert D, Stone JL, Skinner AC, Muir K et al. The relationship between glaucoma medication adherence, eye drop technique, and visual field defect severity. Ophthalmology 2011; 118: 2398-2402.

14 Cronin TH, Kahook MY, Lathrop KL, Noecker RJ. Accuracy and performance of a commercially available Dosing Aid. Br J Ophthalmol 2007; 91: 497-499.

15 Friedman DS, Jampel HD, Congdon NG, Miller R, Quigley HA. The TRAVATAN Dosing Aid accurately records when drops are taken. Am J Ophthalmol 2007; 143: 699-701.

16 Nordmann JP, Baudouin C, Renard JP, Denis P, Lafuma A, Laurendeau $C$ et al. Measurement of treatment compliance using a medical device for glaucoma patients associated with intraocular pressure control: a survey. Clin Ophthalmol 4: 731-739.

17 Ajit RR, Fenerty CH, Henson DB. Patterns and rate of adherence to glaucoma therapy using an electronic dosing aid. Eye (London) 2010; 24: 1338-1343.

18 DiMatteo MR. Variations in patients' adherence to medical recommendations: a quantitative review of 50 years of research. Med Care 2004; 42: 200-209.

19 Haynes RB, McDonald HP, Garg AX. Helping patients follow prescribed treatment: clinical applications. JAMA 2002; 288: 2880-2883.

20 Benner JS, Glynn RJ, Mogun H, Neumann PJ, Weinstein $\mathrm{MC}$, Avorn J. Long-term persistence in use of statin therapy in elderly patients. JAMA 2002; 288: 455-461. 
21 Hermann MM, Bron AM, Creuzot-Garcher CP, Diestelhorst M. Measurement of adherence to brimonidine therapy for glaucoma using electronic monitoring. J Glaucoma 2011; 20: 502-508.

22 Friedman DS, Okeke CO, Jampel HD, Ying GS, Plyler RJ, Jiang $\mathrm{Y}$ et al. Risk factors for poor adherence to eyedrops in electronically monitored patients with glaucoma. Ophthalmology 2009; 116: 1097-1105.

23 McCambridge J, Butor-Bhavsar K, Witton J, Elbourne D. Can research assessments themselves cause bias in behaviour change trials? A systematic review of evidence from solomon 4-group studies. PLoS One 2011; 6: e25223.

24 Cramer JA, Scheyer RD, Mattson RH. Compliance declines between clinic visits. Arch Intern Med 1990; 150: 1509-1510.

25 Gupta R, Patil B, Shah BM, Bali SJ, Mishra SK, Dada T. Evaluating eye drop instillation technique in glaucoma patients. J Glaucoma 2011; 21(3): 189-192.

26 Weis SE, Slocum PC, Blais FX, King B, Nunn M, Matney GB et al. The effect of directly observed therapy on the rates of drug resistance and relapse in tuberculosis. $N$ Engl J Med 1994; 330: 1179-1184.

27 Granstrom PA. Glaucoma patients not compliant with their drug therapy: clinical and behavioural aspects. $\mathrm{Br} \mathrm{J}$ Ophthalmol 1982; 66: 464-470.

28 Collins D. Pretesting survey instruments: an overview of cognitive methods. Qual Life Res 2003; 12: 229-238.

29 Blair E, Burton S. Cognitive-processes used by survey respondents to answer behavioral frequency questions. J Consum Res 1987; 14: 280-288.

30 Broadway D, Miglior S, Myers JS. Fluctuating intraocular pressure. J Glaucoma 2005; 14: 249-251.
31 Svarstad BL, Chewning BA, Sleath BL, Claesson C. The Brief Medication Questionnaire: a tool for screening patient adherence and barriers to adherence. Patient Educ Counsel 1999; 37: 113-124.

32 Rees G, Leong O, Crowston JG, Lamoureux EL. Intentional and unintentional nonadherence to ocular hypotensive treatment in patients with glaucoma. Ophthalmology 2010; 117: 903-908.

33 NICE. Medicines Adherence: Involving Patients in Decisions About Prescribed Medications and Support Adherence. National Collaborating Centre for Acute Care, National Institute for Health and Clinical Excellence: London, UK, 2009.

34 Denis P, Covert D, Realini A. Travoprost in the management of open-angle glaucoma and ocular hypertension. Clin Ophthalmol 2007; 1: 11-24.

35 Dubiner HB, Sircy MD, Landry T, Bergamini MV, Silver LH, Darell Turner F et al. Comparison of the diurnal ocular hypotensive efficacy of travoprost and latanoprost over a 44-hour period in patients with elevated intraocular pressure. Clin Ther 2004; 26: 84-91.

36 Gray TA, Orton LC, Henson D, Harper R, Waterman H. Interventions for improving adherence to ocular hypotensive therapy. Cochrane Database Syst Rev 2009; 15(2): CD006132.

37 McIlraith I, Strasfeld M, Colev G, Hutnik CM. Selective laser trabeculoplasty as initial and adjunctive treatment for openangle glaucoma. J Glaucoma 2006; 15: 124-130.

38 Nagar M, Ogunyomade A, O'Brart DP, Howes F, Marshall J. A randomised, prospective study comparing selective laser trabeculoplasty with latanoprost for the control of intraocular pressure in ocular hypertension and open angle glaucoma. Br J Ophthalmol 2005; 89: 1413-1417. 